\title{
METHODOLOGY FOR ESTIMATING THE DEPENDENCE BETWEEN FORCE AND DEPLACEMENT - A VEHICLE CRASH CASE
}

\author{
Dr Radomir Mijailović * \\ University of Belgrade, Faculty of Transport and Traffic Engineering, Belgrade, Serbia
}

Accident reconstruction software analyzed impact of vehicles considers usage of coefficient of restitution as a known quantity. In practice it is common that numerical value of coefficient of restitution is determined on basis of experience. Presence of error in its evaluation causes an essential error in output results. Thus, it is of exceptional importance to determine more precisely its numerical value. Improved solution of vehicle accident reconstruction software is presented in this paper. To archive that, in this paper was developed methodology which provides such analysis of impact process in which the coefficient of restitution becomes the result, and not input data. We have suggested new methodology for mathematical modeling of function dependence between force and displacement in a collision of vehicles. The compression process was approximated with piecewise linear function. The restitution process was approximated with linear curves. During process of restitution stiffness depends on maximum value of displacements during process of compression. The quality of the coherence between experimental data and mathematical function is quantified by residual sum of squares. Numerical examples are performed by usage of the obtained methodology.

Keywords: methodology, reconstruction, vehicle, coefficient of restitution, error.

\section{INTRODUCTION}

Coefficient of restitution represents a variable which substantially affects the output values which are obtained by analysis of collisions of bodies. Usually mechanical-mathematical models presume cognition of numeric values of coefficient of restitution. In practice, it is common that numerical value of coefficient of restitution is determined on basis of experience. This practice is particularly used in the case of inhomogeneous bodies of complex shape. One of the practical examples is the analysis of impact of vehicles.

There are a few approaches in literature for defining the coefficient of restitution. The usual way is trough usage of characteristic velocities during the impact. In case of direct central collision of two bodies, coefficient of restitution is defined as ratio of absolute values of velocities at the end and the beginning of collision [21].

The coefficient of restitution can be defined using the graph of dependence between force and time. Coefficient of restitution is in this way defined as a ratio of impulse of restitution and impulse of compressive force $[8,15]$.
The coefficient of restitution is a function of velocity immediately prior to the collision. This connection is confirmed by experimental investigations of Zhang and Vu-Quoc [26]. By analysis of experimental results it may be concluded that during the displacement rise time, the influence of impact velocity on function dependence between force and displacement may be neglected. During the period of displacement decrease, the impact velocity has a non-negligible influence on dependence between force and displacement i.e. that curve dependence between force and displacement for a different value of impact velocity can not be obtained only by simple translation of curve graph. That is to say, the increase of impact velocity increases the slope of forcedisplacement curve.

Ambrosio [1] suggests that it is necessary to include the relation between impact forces and displacements in the crash analysis of vehicles. Several software packages are available for the reconstruction of real vehicle accidents. The PC-Crash software is a common commercial tool for reconstructing road accidents [4]. 
It uses a number of important parameters for vehicle model. Most of them are given by default, within the software, and the results of the accident reconstruction are very sensible to their variations. The coefficient of restitution is one of them, used as the input data, and usually taken arbitrary.

Based on currently published papers a necessity may be noticed for upgrade of old and defining new impact models. This is also one of directions of development in dynamics of multibody systems [20]. To that aim, a methodology is developed in this paper which provides such analysis of impact process in which the coefficient of restitution becomes the result, and not input data. We suggest that accident reconstruction software must have database with vehicles functions of dependence between force and displacement.

The incomplete information about vehicle characteristics can cause incapability of accident reconstruction. Determining the adequate functions dependence between force and displacement of vehicles must to represent one of the major tasks in the analysis of impact of vehicles [6]. Their determination influence importantly on reduction of error in the output result of the analysis of impact process.

There are a few approaches in literature for defining the functions dependence between force and displacement of collisions vehicles. Usually mechanical-mathematical model presume that the curve dependence between force and displacement can be described with two linear curves (one curve for compression process and one curve for restitution process) [16]. In this case force can be described in form of multiplication of displacement and appropriate stiffness. The difficulty lays in determining the stiffness during the processes of compression and restitution of vehicles. The approximate expression for the processes of compression may be found in the literature $[8,14]$ :

$$
c=\frac{m_{v} \cdot v_{0}^{2}}{\Delta_{\max }^{2}}
$$

where

$m_{v}$ - vehicle mass,

$v_{0}$ - impact velocity,

$\Delta$ max - maximal displacement of vehicle.

To my knowledge the analytical expression for exact determination of stiffness during the restitution processes is not yet available. Usually in practical defining the previous stiffness was calculated using coefficient of restitution. What is the problem? Usually mechanical-mathematical models presume cognition of numeric values of coefficient of restitution. In practice, it is common that numerical value of coefficient of restitution is determined is based on experience. The error present in determination of the value of coefficient of restitution has a considerable influence on the outcome results of the collision analysis and determination of stiffness during the processes of restitution.

Does the stiffness during the processes of compression have significant correlation with the vehicle mass? The Insurance Institute for Highway Safety has evaluated the crashworthiness of more than 120 new vehicle models. The data indicate that there were no significant correlation between mass, front-end length, and stiffness to structural performance in the offset test [13].

The force displacement curves for the vehicle models can also be approximated as piecewise linear with three regions: before buckling has started, after buckling has started and when the occupant compartment starts to deform [24]. Elmarakbi and $\mathrm{Zu} \mathrm{[5]} \mathrm{presume} \mathrm{that} \mathrm{the} \mathrm{curve}$ dependence between compression force and displacement can be described using piecewise linear functions but with two regions.

Usually authors presume that the force has zero value at the moment when the appropriate displacement has zero value. However, there are other approaches to determining the functions dependence between force and displacement of vehicles. McCoy and Lankarani [12] presume that the force have value greater than zero at the initial moment of vehicle's crash when the displacement has zero value.

Macmillan [11] stated that what is needed is an analytical expression for the force upon displacement curves that satisfy the following criteria:

- it must be simple enough to be manipulated;

- it must satisfy the boundary conditions found in curves from impact tests;

- it must correlate well with known test cases and hence justify its use to predict the outcome over a range of unknown examples;

- it must be capable of representing the behavior of vehicles with different crush char- 
acteristics with changes to a small number of variables.

Stiffness is different for different surfaces of vehicles. One such example is a frontal underride collision [2].

The experimental data for the crash tests may be found in literature. Most of the tests were made at about $60 \mathrm{~km} / \mathrm{h}[7,27]$. It also can be found papers $[9,22,25]$ which presented the experimental data for the same vehicle at different impact velocity.

In literature great attention has been dedicate to dynamic behavior of vehicles $[3,17,18]$.

Based on currently published papers I have concluded that there is necessity for defining new functions dependence between force and displacement of vehicles.

This paper's objective is to suggest new methodology for mathematical modeling of function dependence between force and displacement in a collision of vehicles. This methodology and function dependence between force and displacement should be simple enough for practical implementation. The purpose of the methodology is reduction of error in the output results of the analysis of vehicle impact process too. The advantage of this methodology is also its easy and fast applicability. From the economic point of view this means lower implementation costs.

Analyzing literature it can be noticed that the case of described previous function with two linear curves is often used. Analyzing experimental data, we have concluded that the linear approximation does not describe graph of dependence between force and displacement on satisfactory way. Therefore, we have approximated the compression process with piecewise linear functions. The restitution process was approximated with linear curves. During process of restitution stiffness (coefficients of proportionality) depends on maximum value of displacements during

$$
F_{k, n}=\left\{\begin{array}{lll}
c_{1} \cdot \Delta & \text { for } 0 \leq \Delta \leq \Delta_{A_{1}} \\
c_{1} \cdot \Delta_{A_{1}}+c_{2} \cdot\left(\Delta-\Delta_{A_{1}}\right) & \text { for } & \Delta_{A_{1}} \leq \Delta \leq \Delta_{A_{2}} \\
c_{1} \cdot \Delta_{A_{1}}+c_{2} \cdot\left(\Delta_{A_{2}}-\Delta_{A_{1}}\right)+c_{3} \cdot\left(\Delta-\Delta_{A_{2}}\right) & \text { for } & \Delta_{A_{2}} \leq \Delta \leq \Delta_{A_{3}} \\
\cdots & & \ldots \\
c_{1} \cdot \Delta_{A_{1}}+c_{2} \cdot\left(\Delta_{A_{2}}-\Delta_{A_{1}}\right)+\ldots+c_{n} \cdot\left(\Delta-\Delta_{A_{n-1}}\right) & \text { for } & \Delta_{A_{n-1}} \leq \Delta \leq \Delta_{A_{n}}
\end{array}\right.
$$

where

$\Delta_{\text {A., }}(\mathrm{i}=1, \ldots, \mathrm{n}-1)-$ maximum value of displacements during process of compression for region "i" (experimental data),

ci, $(\mathrm{i}=1, \ldots, \mathrm{n})-$ coefficients of proportionality process of compression. The quality of the coherence between experimental data and mathematical function is quantified by residual sum of squares.

\section{METHODOLOGY FOR ESTIMATING THE DEPENDENCE BETWEEN FORCE AND DISPLACEMENT}

\section{The function force-displacement}

The function dependence between force and displacement is determined by the following expression:

$$
F=\left\{\begin{array}{lll}
F_{k} & \text { for } & \dot{\Delta} \geq 0 \\
F_{r} & \text { for } & \dot{\Delta} \leq 0
\end{array}\right. \text {, }
$$

where

$F_{k}$ - the function dependence between compression force and displacement,

$F_{r}$ - the function dependence between restitution force and displacement,

$\dot{\Delta}$ - displacement of vehicle.

In this paper the function dependence between compression force and displacement of vehicles is analyzed for piecewise linear functions with one and more than one regions ( $\mathrm{n}$ regions) (Figure 1).

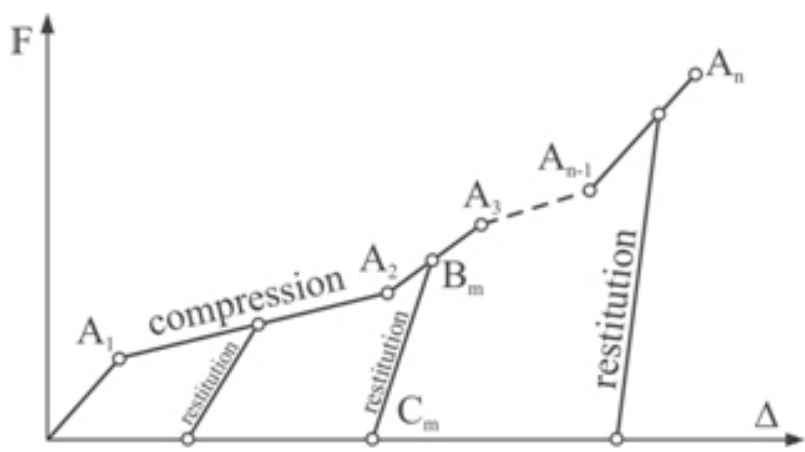

Figure 1. Dependence between force and displacement

Function of dependence between compression force and displacement is given by expression:

during process of compression for region " $i$ ". Coefficient of proportionality $\left(c_{1}\right.$ for $\left.i=1\right)$ in literature is known as the coefficients of stiffness. Therefore, in the following text we will use notion stiffness for $c i$. 
It is necessary to define following unknown parameters ci and $\Delta_{\mathrm{A}, \mathrm{i}}$.

Experiment data are necessary to obtain the function dependence between force and displacement. In that sense experiment consists of impact of vehicles into an absolutely rigid barrier. To gain better results experiment must be repeated for different values of impact velocity $[9,10,22,23,24,25]$. The experiment may be realized through crash test or by finite element method based computer program.

The advantage of this methodology is also its easy and fast applicability. From the economic point of view this means lower implementation costs. Respecting legal terms, all new car models must pass crash tests before they are sold. But legislation provides a minimum statutory standard of safety for new vehicles, it is the aim of Euro NCAP to encourage manufacturers to exceed these minimum requirements [28]. Crash tests must be realized for all vehicles. Addition economic investment is necessary for process of crash test results. Vehicle manufacturer develop finite element models for their vehicles. Their addition assignment should be utilization of the vehicle finite element model using computer program. Minimum addition economic investment is also requested.

\section{The residual sum of squares}

The quality of the coherence between experimental curves and mathematical functions is quantified by residual sum of squares. Why was chosen just residual sum of squares? The function of dependence between compression force and displacement done by the minimization of a residual sum of squares also has minimum error using it in determination work during compression process. A minimal error in determining the work also results in a minimal error in determining coefficient of restitution.

Residual sum of squares (RSS) measures the deviations of experimental from their predicted values:

$$
R S S=\sum_{i=1}^{n k}\left(F_{k, i}-F_{k, \exp , i}\right)^{2}
$$

\section{where}

$F_{k, \text { exp }}, i$ is the ith experimental value of the variable to be predicted, and $F_{k, i}$ is the predicted value of $F_{k, \text { exp }, i}$

$n_{k}$ - represents number of experimental values.

\section{The determination of unknown parameters}

The determination of unknown parameters was performed by the minimization of a residual sum of squares. We concluded that the analytical solution of minimization of the function (4) can't be determined for $n \geq 1$ (3) by application of some optimization methods [17]. Therefore, the unknown parameters (ci, $\Delta_{A, i}$ ) should be numerically determined.

The restitution process is significantly shorter than compression process. Using more complex functions error would not be significantly reduced. On the other hand they would be more complicated for practical implementation. Therefore, the restitution process was approximated with linear curve (Figure 1). Slopes of restitution lines are a function of maximum value of displacement during process of compression ( $\Delta_{\mathrm{Bm}}$ ).

Functions of dependence between restitution forces and displacement are given by expression:

$$
F_{r, m}=F_{k}\left(\Delta_{B_{m}}\right)-b_{m} \cdot\left(\Delta_{B_{m}}-\Delta\right)
$$

where

$\Delta_{\mathrm{Bm}}$ - maximum value of displacements during process of compression,

$b m$ - coefficients of proportionality (stiffness) during process of restitution for $\Delta_{\mathrm{Bm}}$.

The coefficients of proportionality during process of restitution $(\mathrm{bm})$ depend of maximum values of displacements during process of compression $\left(\Delta_{\mathrm{Bm}}\right)$. Therefore, we have defined the coefficients of proportionality $\mathrm{bm}$ as function dependence of maximum value of displacements during process of compression $\left(\Delta_{B m}\right)$.

The coefficients of proportionality during process of restitution (bm) can be determined from the conditions: during restitution process work determined by experimental data (Ar,m,exp) is equal to work determines using approximate functions (5) $\left(A_{r, m, \text { approx }}\right)$ :

$$
A_{r, m, \exp }=A_{r, m, \text { approx }}
$$

Numerical value of work $A_{r, m, \exp }$ can be determined by using experimental data for the same vehicle characteristics and for different values of impact velocities. The analysis output data are two numerical values: $\Delta_{B m}$ and $A_{r, m, \exp }$. 
Numerical value of work Ar,m,approx is given in following form:

$$
A_{r, m, \text { approx }}=\int_{\Delta_{B_{m}}}^{\Delta_{C_{m}}} F_{r, m} d \Delta .
$$

Displacement of vehicle in the instant of separation $\left(\Delta_{c m}\right)$ may be determined from the condition:

$$
F_{r, m}\left(\Delta_{C_{m}}\right)=0
$$

The displacement $\Delta_{c m}$ can be determined by substitution of (5) into (8):

$$
\Delta_{C_{m}}=\Delta_{B_{m}}-\frac{F_{k}\left(\Delta_{B_{m}}\right)}{b_{m}}
$$

Finally, stiffness bm can be determined by applying equations (5), (6), (7) and (9):

$$
b_{m}=-\frac{\left[F_{k}\left(\Delta_{B_{m}}\right)\right]^{2}}{2 \cdot A_{r, m, \exp }}
$$

The stiffness $b m$ depends on the displacement (10) $\Delta_{B m}$. The next step of the analysis should be finding the equation that will approximate the dependence between $\mathrm{bm}$ and $\Delta_{\mathrm{Bm}}$ on the best way. Previous equation should be determined using some software for curve fitting analysis. The curve fitting analysis is necessary because the experimental data have results for the different (but not all) values of impact velocity. For example, we may be having experimental data for impact velocities: 10, 20, 30, 50, 70 and $100 \mathrm{~km} / \mathrm{h}$. Accident reconstruction software may be needed the stiffness bm that should be calculated using experimental data for example for impact velocity of $57 \mathrm{~km} / \mathrm{h}$.

Finally, the methodology for mathematical modeling of function dependence between force and displacement is given in following form:

- collecting experimental data for different values of impact velocity;

- the determination of unknown parameters $\left(c i, \Delta_{A, j}\right)$ by the minimization of a residual sum of squares;

- to determine number of regions ( $n$ ) for piecewise linear function of dependence between compression force and displacement;

- to write function of dependence between compression force and displacement (3);

- the determination of works $A_{r, m, \exp }$ for different values of $\Delta_{B m}$;
- the determination of stiffness bm for different values of $\triangle B m$;

- to determine equation that approximate dependence between bm and $\mathrm{Bm}$ on the best way;

- to write function of dependence between restitution force and displacement (5);

- finally, we can write the function dependence between force and displacement (2).

Vehicle doesn't have the same functions dependence between force and displacement (2) for different point of collisions vehicles. For example, frontal, rear and side vehicle area does not have the same values of stiffness. That is why NCAP is performing different crash tests. Therefore, suggested methodology must be applied for different vehicle's areas.

\section{RESULTS ANALYSIS}

The first key question that arises is: does linear approximation describe dependence between compression force and displacement better than piecewise linear function?

The determination of unknown parameters was performed using experimental data given in papers $[9,10,22,23,24,25]$. The experimental data from previous papers was derived using the case of direct central impact of vehicles into an absolutely rigid barrier.

Quality comparison between two piecewise linear functions with subsequent number of regions (i.e. $n$ and $n+1$ ) may be presented by comparing their RSS relative discrepancy:

$\delta R S S_{n=i \wedge n=i+1}=\left|\frac{R S S_{i+1}-R S S_{i}}{R S S_{i+1}}\right| \cdot 100, \%$

The same calculation was used for comparing two functions with $n=1$ where stiffness obtained using equations (1) and (4):

$$
\begin{aligned}
\delta R S S_{n=1(E q .1 \wedge E q .4)}= \\
\quad=\left|\frac{R S S_{(E q .1)}-R S S_{(E q .4)}}{R S S_{(E q .1)}}\right| \cdot 100, \%
\end{aligned}
$$

The relative discrepancy of the compression force for different values of regions was determined based on the experimental data obtained from 14 vehicles (Table 1). Results of the analysis show that the minimal relative discrepancies are appeared between piecewise linear functions dependence of compression force upon displacement with three and four regions. 
The relative discrepancy $\delta \mathrm{RSSn}=3 \wedge \mathrm{n}=4$, for the most vehicles, tend to zero. The other relative discrepancies have bigger values. Looking at obtained results it may be noticed that the rela- tive discrepancy $\delta \mathrm{RSSn}=2{ }_{\wedge} \mathrm{n}=3$ is between 1.5 and $28 \%$. The relative discrepancy $\delta$ RSSn $=1$ $\mathrm{n}=2$ is between 21.6 and $200.4 \%$. The relative discrepancies for $n_{\geq} 4$ tend to zero.

Table 1. Relative discrepancy ( $R S S$ ) of the compression force for different values of regions and for various vehicle models

\begin{tabular}{|c|c|c|c|c|}
\hline \multirow{2}{*}{ Vehicle } & $\delta \mathrm{RSSn}=1(\mathrm{Eq} .1 \mathrm{Eq} .4)$ & $\delta \mathrm{RSSn}=1 \wedge \mathrm{n}=2$ & $\delta \mathrm{RSSn}=2 \mathrm{n}=3$ & $\mathrm{RSSn}=3 \wedge \mathrm{n}=4$ \\
\hline & \multicolumn{4}{|c|}{$\%$} \\
\hline Chrysler Neon [24] & 5.7 & 42.4 & 23.3 & 1.1 \\
\hline Dodge Caravan [24] & 51.2 & 21.6 & 28 & 0.2 \\
\hline Ford Escort [9] & 82.8 & 166.1 & 14.9 & 0 \\
\hline Ford Escort [22] & 11.4 & 122.1 & 3.7 & 0.1 \\
\hline Ford Explorer [24] & 23 & 169.9 & 1.5 & 2.7 \\
\hline Ford Taurus [24] & 25.1 & 446.8 & 6 & 0 \\
\hline Geo Metro [24] & 1.2 & 25 & 11.9 & 0 \\
\hline Honda Accord [24] & 94.5 & 200.4 & 23.4 & 0 \\
\hline Vehicle 1 [23] & 3.1 & 87.2 & 18.6 & 0 \\
\hline Vehicle 2 [23] & 0.7 & 104.6 & 8.9 & 0 \\
\hline Vehicle 3 [23] & 9.4 & 90.2 & 6.8 & 0 \\
\hline Vehicle 4 [23] & 8.4 & 142.9 & 4.2 & 0 \\
\hline Vehicle 5 [23] & 0.1 & 102.8 & 6.7 & 0 \\
\hline Yugo [10] & 492.1 & 175.9 & 4.5 & 0 \\
\hline
\end{tabular}

The approximate expression for the compression stiffness (1) is often used in practice. By analysis of $\delta$ RSSn=1(Eq.1 $\wedge$ Eq.4) (Table 1) it can be concluded that this expression doesn't have satisfactory results.

Therefore, we propose to use piecewise linear functions dependence between compression force and displacement with three regions.

In the following text we will present results of usage of methodology that we have proposed. We have used experimental data obtained for Ford Escort [22].

Compression stiffness (ci) and displacement $\left(\Delta_{A, j}\right)$ for linear functions dependence between compression force and displacement with three regions are:

$$
\begin{aligned}
& c_{1}=1034 \mathrm{kN} / \mathrm{m} ; \Delta_{A_{1}}=0.16 \mathrm{~m} ; \\
& c_{2}=299 \mathrm{kN} / \mathrm{m} ; \Delta_{A_{2}}=0.43 \mathrm{~m} ; \\
& c_{3}=38 \mathrm{kN} / \mathrm{m} ; \Delta_{A_{3}}=1.23 \mathrm{~m} .
\end{aligned}
$$

$$
\begin{aligned}
& F= \begin{cases}1034 \cdot \Delta & \text { for } \quad \dot{\Delta} \geq 0 \wedge 0 \leq \Delta \leq 0.16 \\
165.44+299 \cdot(\Delta-0.16) & \text { for } \quad \dot{\Delta} \geq 0 \wedge 0.16 \leq \Delta \leq 0.43 \\
246.17+38 \cdot(\Delta-0.43) & \text { for } \dot{\Delta} \geq 0 \wedge 0.43 \leq \Delta \leq 1.23 \\
F\left(\Delta_{B}\right)-\left(50036.905+63960.156 \cdot \Delta_{B}^{10.599}\right) \cdot\left(\Delta_{B}-\Delta\right) & \text { for } \dot{\Delta} \leq 0\end{cases} \\
& F, k N \quad \Delta, \Delta_{B}, m,
\end{aligned}
$$

The stiffness bm for Ford Escort were approximated using the results (14) by curve fitting analysis:

$b=50036.905+63960.156 \cdot \Delta_{B}^{10.599}, k N / m$

By applying expressions (3), (5), (13) and (15) the function dependence between force and displacement (2) for Ford Escort obtains its final form: 
where $\Delta_{B}$ is the maximum value of displacements during process of compression.

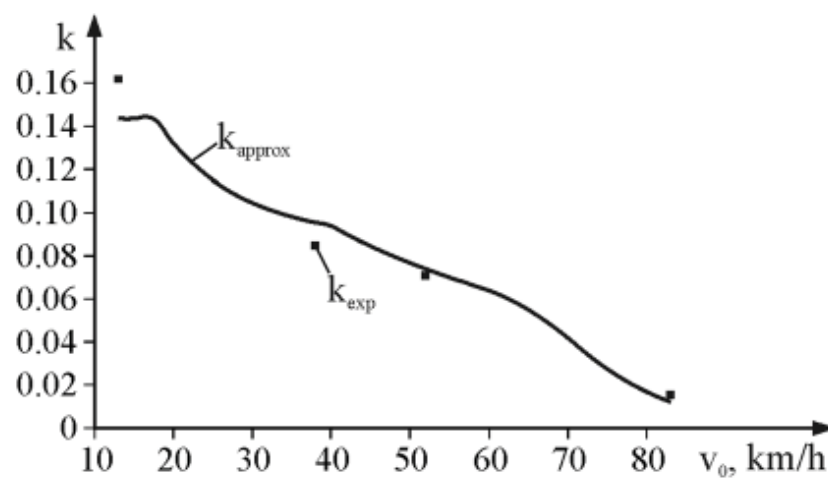

Figure 2. Dependence between coefficient of restitution and impact velocities for Ford Escort

Consider an accident where Ford Escort has crashed into an absolutely rigid barrier. The software packages for the reconstruction of real vehicle accidents require usage coefficient of restitution as the input data. The purpose of the model presented in this paper is reduction of error in the output results of analysis of vehicle impact process. The next key question that arises is: how big is the error that is made using the model presented in this paper? Therefore, coefficient of restitution ( $\left.k_{\text {approx }}\right)$ of impact of Ford Escort into an absolutely rigid barrier is calculated using the methodology for mathematical modeling of function dependence between force and displacement presented in this paper. The results were presented on the Figure 2 . The coefficient of restitution calculated using appropriate experimental data $\left(k_{\text {exp }}\right)$ was also presented on the Figure 2. By analyzing Figure 2 it can be concluded that the errors have minimal values. The absolute error of coefficient of restitution has value in the range from 0.003 to 0.018 .

\section{CONCLUSIONS}

The model developed in this paper which provides such analysis of impact process in which the coefficient of restitution becomes the result, and not input data. In this paper was developed methodology that can be used in analysis of impact process in which the coefficient of restitution becomes the result, and not input data. In this paper is suggested new methodology for mathematical modeling of function dependence between force and displacement in a collision of vehicles. This methodology and function dependence between force and displacement should be simple enough for practical implementation. The purpose of the methodology is reduction of error in the output results of the analysis of vehicle impact process too. The advantage of this methodology is also its easy and fast applicability. From the economic point of view this means lower implementation costs.

Further investigations may be directed towards establishing an analytical model for analysis of three dimensional vehicles collisions with the coefficient of restitution as the end result. Since the three dimensional collision produces complex motion, future model should take into account the friction. Such analysis of collision processes would contribute to the analysis of traffic accidents.

\section{ACKNOWLEDGEMENT}

The research work was supported by the Ministry of Science and Technological Development of the Republic of Serbia (Grant No. 36010).

\section{REFERENCES}

1) Ambrosio, J. (2005). Crash analysis and dynamical behaviour of light road and rail vehicles. Vehicle System Dynamics, Vol. 43, No. 6-7, pp. 385-411

2) Boggessa, B.M., Morrb, D.B., Petermanb, E.K., Wiechel, J.F. (2010). Experimental evaluation of underride analysis techniques and empirical validation of a new analytical technique. Accident Analysis and Prevention, No. 42, pp. 140-152

3) Demić, M. (2005). A contribution to design of semiactive vehicle suspension system. Journal of Applied Engineering Science (Istraživanja i projektovanja za privredu), 9, pp. 7-16

4) Depriester, J.P., Perrin, C., Serre, T., Chalandon, S. (2006). Comparision of several methods for real pedestrian accident. (available on-line: www.mathlab.mtu.edu)

5) Elmarakbi, A., Zu, J. (2007). Mathematical modelling of a vehicle crash with emphasis on the dynamic response analysis of extendable cubic nonlinear dampers using the incremental harmonic balance method. Proc. IMechE Part D: J. Automobile Engineering, Vol. 221, pp. $143-156$

6) Harmati, I.A., Rovid, A., Varlaki, P. (2010). Application of LPV Type Force Model in Vehicle Crash Dynamics. Proceedings of the 
9th WSEAS International Conference on Applications of computer engineering

7) Huibers, J., de Beer, E. (2001). Curent front stiffness of European vehicles with regard to compatibility. International Technical Conference on the Enhanced Safety of Vehicles (ESV), Amsterdam, The Netherlands

8) Janković, A., Simić, D. (1996). Vehicle safety, monograph. DSP-mecatronic, Kragujevac

9) Kerkhoff, J.F., Husher, S.E., Varat, M.S., Busenga, A.M., Hamilton, K. (1993). An investigation into vehicle frontal impact stiffness, BEV and repeated testing for reconstruction. Society of Automotive Engineers International Congress, Detroit, Michigan, SAE Paper 930899

10) Kostić, S., Bogićević, D., Lalić, Z. (2007). Improving of methods for determination collision speed of vehicles based on their deformations. XXI International JUMV Automotive Conference Science and Motor Vehicles, $\mathrm{Pa}$ per NMV0720S

11) Macmillan, R.H. (1983). Dynamics of vehicle collisions, Interscience Enterprises Ltd

12) McCoy, M.L., Lankarani, H.M. (2006). Determination of the crush stiffness coefficients of a typical aftermarket frontal protective guard used in light trucks and vans with comparisons between guard stiffness and frontal vehicle crush coefficients. Proceedings of the Institution of Mechanical Engineers, Part D: Journal of Automobile Engineering, Vol. 220, pp. $1073-1084$

13) Nolan, J.M., Lund, A.K. (2001). Frontal offset deformable barrier crash testing and its effect on vehicle stiffness. International Technical Conference on the Enhanced Safety of Vehicles (ESV), Amsterdam, The Netherlands

14) Nusholtz, G.S., Xu, L., Shi, Y., Domenico, L.D. (2005). Vehicle mass, stiffness and their relationship. 19th ESV, Paper number 050413

15) Oztas, A.G. (1999). Application of Impulse Momentum Theory to Vehicle Collisions. Turkish Journal of Engineering and Environmental Science, 23, pp. 455-464

16) Pawlus, W., Karimi, H.R., Robbersmyr, K.G. (2011). Mathematical modeling of a vehicle crash test based on elasto-plastic unloading scenarios of spring-mass models. The International Journal of Advanced Manufacturing Technology, 55, pp.:369-378
17) Radisavljević, M., Demić, M. (2004). A contribution to car body design of a passanger motor vehicle through improved vibro-acoustic parameters. Journal of Applied Engineering Science (Istraživanja i projektovanja za privredu), 6, pp. 25-34

18) Sekulić, D., Dedović, V. (2008). Simulation of the oscillatory behavior of buses equipped with a classic and active suspension system. Journal of Applied Engineering Science (Istraživanja i projektovanja za privredu), 20, pp. 23-32

19)Šelmić, R., Cvetković, R., Mijailović, R. (2006). Optimization of cross-section in structures. monograph, The Faculty of Transport and Traffic Engineering, Belgrade

20) Schiehlen, W. (2007). Research trends in multibody system dynamics. Multibody System Dynamic, 18: 3-13

21) Stronge, W.J. (2004). Impact mechanics. Cambridge University Press

22) Steffan, H., Geigl, B.C., Moser, A., Hoschopf, H. (1998). Comparison of 10 to $100 \mathrm{~km} / \mathrm{h}$ rigid barrier impacts. Paper No. 98-S3-P-12, (www-nrd.nhtsa.dot.go)

23) Subramaniam, K., Verma, M., Nagappala, R., Tedesco, R., Carlin, L. (2007). Evaluation of stiffness matching concepts for vehicle safety improvement, Enhanced Safety of Vehicles Conference, Lyon, France, Paper Number 07-0112

24) Van der Zweep, C.D., Jenefeldt, F., Thomson, R. (2002). Improvement of vehicle crash compatibility through the development of crash test procedure. Project number GRD2-2001-50083, 1998-2002

25) Varat, M.S., Husher, S.E. (2000). Vehicle impact response analysis through the use of acceleration data. SAE Tech. Paper 200001-0850

26) Zhang, X., Vu-Quoc. L. (2002). Modeling the dependence of the coefficient of restitution on the impact velocity in elasto-plastic collisions, International Journal of Impact Engineering, 27, pp. 317-341.

27) www.nhtsa.dot.gov

28) www.euroncap.com

Paper sent to revision: 17.02.2012.

Paper ready for publication: 23.03.2012. 